\title{
4 Estudo comparativo: Ultrassom e drenagem linfática manual associando THERAPY TAPING $®$ na redução da circunferência abdominal
}

\author{
Comparative study: Ultrasound and manual \\ lymphatic drainage associating THERAPY \\ TAPING $®$ in reducing waist circumference
}

FisiSenectus. Unochapecó Ano 3, n. 2 - Jul/Dez. 2015 p. $59-67$

Jéssica Lopes de Souza. jehssicah@hotmail.com Acadêmica do curso de Fisioterapia da Universidade Braz Cubas - UBC - Mogi das Cruzes - SP.

Pedro Vinicius Nabeiros Martins. pedronabeiros@hotmail.com Acadêmico do curso de Fisioterapia da Universidade Braz Cubas - UBC - Mogi das Cruzes - SP

Ricardo Mingorance. rimingorance@hotmail.com Acadêmico do curso de Fisioterapia da Universidade Braz Cubas - UBC - Mogi das Cruzes - SP.

Márcia Regina Pinez Mendes. marcia.pinez@brazcubas.br

Fisioterapeuta, Mestre em Educação e Desenvolvimento Humano - Unicamp; Profa do Curso de Fisioterapia da Universidade Braz Cubas - UBC - Mogi das Cruzes - SP.

Laila Moussa. IImoussa@hotmail.com

Fisioterapeuta, Mestre em Ciências do Movimento - UNG; Profa do Curso de Fisioterapia da Universidade Braz Cubas - UBC - Mogi das Cruzes - SP.

\section{Resumo}

Introdução: 0 público feminino frequentemente submete-se à Fisioterapia Dermatofuncional com a expectativa de resultados para seus problemas relacionados à estética, como a lipodistrofia localizada que é o acúmulo regional de tecido adiposo. Objetivos: Comparar os efeitos do ultrassom e drenagem linfática manual associado ao Therapy Taping ${ }^{\circledR}$ na redução da circunferência abdominal. Materiais e métodos: Estudo realizado com seis indivíduos do gênero feminino, divididos em dois grupos, um submetido ao ultrassom associado à drenagem linfática manual e o outro submetido ao ultrassom, drenagem linfática manual e Therapy Taping ${ }^{\circledR}$. Para verificar o efeito do Therapy Taping ${ }^{\circledR}$ a aplicação foi em forma de "teia", por meio do direcionamento da bandagem no sentido do retorno linfático. No total foram 12 atendimentos, duas vezes por semana para cada indivíduo, considerando a média de 60 minutos para cada sessão. A perimetria foi realizada em: nível da cicatriz onfálica, $3 \mathrm{~cm}$ acima e $3 \mathrm{~cm}$ abaixo, avaliadas antes da $1^{\mathrm{a}}$ sessão e após a $12^{\mathrm{a}}$. Resultados: Com o protocolo foram constatados resultados nas reduções em centímetros da região da cicatriz onfálica e acima. Quando comparados os resultados de redução a partir de porcentagem, o grupo 2 apresentou redução superior apenas em área de cicatriz onfálica. Conclusão: concluiu-se que o Therapy Taping ${ }^{\circledR}$ não influenciou na redução da circunferência abdominal. A amostra foi pequena, o que pode ter influenciado nos resultados. Saliente-se que mediante pesquisas realizadas não foram encontrados estudos do tipo experimental intervencionista com o tema proposto, sendo este um trabalho inovador, ampliando o campo de ideias para composição de novos estudos

Palavras-chave:

Lipodistrofia. Manipulações Musculoesqueléticas. Circunferência Abdominal. Fisi ene CtUS 


\section{Abstract}

Introduction: The female audience Dermatofuncional Physiotherapy with the expected results for its aestheticrelated problems such as localized lipodystrophy which is the regional accumulation of adipose tissue. Objectives: To compare the effects of ultrasound and manual lymphatic drainage associated with Therapy Taping ${ }^{\circledR}$ in reducing waist circumference. Methods: Study with 6 female subjects divided into two groups, one submitted to ultrasound associated with manual lymphatic drainage. The other subjected to ultrasound, manual lymphatic drainage and Therapy Taping ${ }^{\circledR}$. To check the effect of Therapy Taping ${ }^{\circledR}$ the application was in a "web" through the banding direction towards the lymphatic return. A total of 12 sessions, twice a week for each individual and average of 60 minutes for each session. Perimetry was taken at: level onfálica scar, 3 $\mathrm{cm}$ above and $3 \mathrm{~cm}$ below assessed before the 1 st session and after the 12 th. Results: With the protocol was observed results in reductions in centimeters of onfálica scar region and above. When comparing the results of reduction by percentage, group 2 showed superior reduction only in onfálica scar area. Conclusion: With this it was concluded that the Therapy Taping ${ }^{\circledR}$ did not influence the reduction of waist circumference. The sample was small, which may have influenced the results. It should be noted that through research conducted were not found interventional experimental study designs with the theme, which is an innovative work expanding the field of ideas for composition of new studies.

\section{Keywords:}

Lipodystrophy. Musculoskeletal Manipulations. Abdominal Circumference.

\section{Introdução}

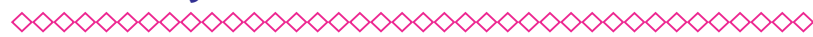

Grande parte do público feminino recorre a métodos e técnicas da área da Fisioterapia Dermato-Funcional com o objetivo de obter resultados para seus problemas relacionados à saúde e estética, como é o caso da Lipodistrofia ${ }^{1}$.

Lipodistrofia é o acúmulo excessivo de gordura localizada, caracterizado por um distúrbio no metabolismo do tecido adiposo formado por células denominadas adipócitos, responsáveis por armazenar a gordura e que apresentam a capacidade de aumentar ou diminuir seu volume de acordo com a quantidade de triglicerídeos em seu interior ${ }^{2}$.

A lipodistrofia localizada é o acúmulo regional de tecido adiposo. A localização do acúmulo varia de acordo com o sexo: homens com predomínio na região do abdome e mulheres em regiões femoroglúteas. Na mulher a localização pode ser influenciada por seu biótipo, classificada como ginóide, acúmulo na metade inferior do corpo, ou andróide, na metade superior ${ }^{3}$

O acúmulo de gordura na região do abdome vem sendo descrito como o que oferece maior risco para a saúde dos indivíduos. Pesquisas comprovam que o acúmulo, mesmo em não obesos, é prejudicial à saúde ${ }^{4,5}$. Tem-se observado uma preocupação com o tipo de depósito de distribuição de gordura, uma vez que esta se relaciona com o prognóstico de risco para a saúde ${ }^{6}$. A Organização Mundial de Saúde (OMS) estabelece como ponto de corte para risco cardiovascular o aumento da medida da circunferência abdominal igual ou superior a 94 cm em homens e $80 \mathrm{~cm}$ em mulheres caucasianas ${ }^{7}$.

Usado na medicina desde a década de 50 para diagnóstico por imagem, na dermatologia o ultrassom (US) possui finalidade terapêutica: tratamento da celulite e gordura localizada8. As ondas ultrassônicas dependem de uma variedade de fatores para atingir a região determinada, tais como: intensidade, frequência, regime de pulso, área do transdutor, tempo de aplicação, técnica de aplicação e agente de acoplamento ${ }^{9}$

Existem dois regimes de pulso comumente empregados na prática clínica do ultrassom terapêutico: o contínuo e o pulsado ${ }^{9}$, sendo necessária a utilização de gel como substância de acoplamento. A onda será captada pelos aminoácidos e proteínas fazendo oscilar estes elementos, mobilizando-os do meio intersticial para o linfático e facilitando a sua absorção ${ }^{1}$.

O ultrassom contínuo apresenta efeito térmico dominante e o pulsado apresenta efeito mecânico dominante, sendo o contínuo com efeito mecânico 
Jéssica Lopes de Souza, Pedro Vinicius Nabeiros Martins, Ricardo Mingorance, Márcia Regina Pinez Mendes e Laila Moussa

maior que o pulsado, capaz de produzir efeito térmico, sendo este o efeito que predomina. No modo pulsado, como não há produção de calor (atérmico), há somente o efeito mecânico, porém inferior ao contínuo².

O ultrassom terapêutico (UST) tem sido usado extensivamente nas últimas décadas para terapias físicas e como meio promotor de permeação cutânea de fármacos. O uso do UST como facilitador na absorção cutânea é conhecido como fonoforese ${ }^{5}$.

No que se refere à linfa, há total concordância que a massagem aumenta seu fluxo. Os linfonodos estão espalhados ao longo do corpo no trajeto dos vasos linfáticos, podendo ser encontrados em maior quantidade na virilha, axila, região cervical, tórax e mesentério ${ }^{10}$. 0 sistema linfático representa uma via auxiliar de drenagem do sistema venoso. Os líquidos provenientes do interstício são devolvidos ao sangue por meio da circulação linfática, que está intimamente ligada à circulação sanguínea e aos líquidos teciduais.

Quando há uma melhora da circulação da linfa, há também uma maior oxigenação dos tecidos do corpo, o que melhora o aspecto da gordura localizada. A drenagem linfática manual é um recurso terapêutico que se caracteriza por manobras suaves e rítmicas sobre os linfonodos e a pele, estimulando a circulação linfática, melhora a condição dos tecidos e promove a desintoxicação ${ }^{11}$.

Um tipo de agente físico recentemente incorporado na Fisioterapia é o Método Therapy Taping ${ }^{\circledR}$ criado pelo Fisioterapeuta Nelson Morini Junior que é uma técnica de aplicação de bandagens elásticas. Essas bandagens foram desenvolvidas para se adaptarem da melhor forma à pele humana, os resultados clínicos são explicados teoricamente pelos estímulos táteis gerados pela bandagem nos receptores cutâneos, que ocasionam uma pequena elevação da pele e o aumento do espaço intersticial, favorecendo a reabsorção do edema e a diminuição da pressão local. O pensamento do método está ligado principalmente a difundir o Conceito de Estimulação Tegumentar, que permite nova visão nos atendimentos daqueles que buscam melhor qualidade de vida para seus pacientes, bem como, melhora funcional daqueles que utilizam a bandagem elástica ${ }^{12}$.

Cada marca de bandagem possui constituições físicas diferentes umas das outras sendo a bandagem Therapy Tex do método Therapy Taping® um tecido constituído por fibras de algodão (100\%), com microfios de elastano e cola adesiva corporal de acrílico, o que torna a bandagem hipoalergênica, sendo contraindicada para todas as pessoas que possam ter algum problema na pele, como: dermografia, escamações na pele, pontos cirúrgicos, cicatrizes recentes, psoríase, bolhas, desidrose, tumor, verruga, entre outros. Ao indicar a bandagem como complemento do tratamento, algumas áreas devem ser observadas com especial atenção para não desenvolver irritação na pele em razão de serem áreas mais finas e sensíveis, como as áreas da região abdominal| ${ }^{13}$.

Nesse sentido, este estudo tem como objetivo comparar os efeitos do ultrassom e da drenagem linfática manual associada ao Therapy Taping® na redução da circunferência abdominal, proporcionando, então, maiores informações à áerea da Fisioterapia Dermatofuncional.

\section{Materiais e Métodos}

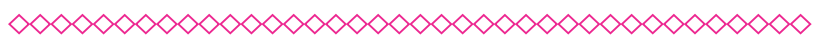

Trata se de um estudo do tipo experimental intervencionista, realizado por meio da seleção de seis indivíduos que preencheram os critérios de inclusão estabelecidos e que aceitaram participar do estudo. Este estudo foi aprovado pelo Comitê de Ética e Pesquisa, sob o parecer n 053/15.

Foram selecionados seis indivíduos que atenderam aos seguintes critérios de inclusão: circunferência abdominal superior a $80 \mathrm{~cm}$, sexo feminino, entre 20 e 30 anos, sedentárias, estudantes da Universidade Braz Cubas.

Foram adotados como critérios de exclusão: indivíduos com circunferência abdominal menor que $80 \mathrm{~cm}$, gestantes, indivíduos com neoplasia, inflamação aguda, feridas abertas na região de aplicação da técnica, alérgicos ao material do Therapy Taping ${ }^{\circledR}$, usuários de medicamentos ansiolíticos, diuréticos, moderadores de apetite e portadores de marca-passo cardíaco. 
No momento da admissão, os indivíduos receberam informações sobre a pesquisa e responderam a um questionário, contendo dados pessoais e histórico social, onde foram inseridas as informações das perimetrias obtidas.

Para medição da circunferência abdominal as voluntárias foram posicionadas em ortostatismo e a perimetria foi feita com uma fita métrica flexível e inelástica, tomando-se o cuidado para não haver compressão dos tecidos. A perimetria foi realizada em três localizações distintas: nível da cicatriz onfálica, $3 \mathrm{~cm}$ acima da cicatriz onfálica e $3 \mathrm{~cm}$ abaixo da cicatriz onfálica ${ }^{8}$. Foram avaliadas antes da $1^{\mathrm{a}}$ sessão de tratamento e após a $12^{\mathrm{a}}$ sessão.

Foram realizados 12 atendimentos, com periodicidade de duas vezes por semana para cada indivíduo, com duração de 60 minutos para cada sessão, no período entre novembro e dezembro de 2015, na Clínica Escola de Fisioterapia da Universidade Braz Cubas, sob a supervisão de um fisioterapeuta responsável.

0 estudo contou com dois grupos de pacientes com três integrantes em cada grupo. 0 grupo 1 (G1) foi submetido ao ultrassom com frequência padronizada em 3 Mhz no modo contínuo e intensidade variando de $0,86 \mathrm{w} / \mathrm{cm} 2$ a $1,5 \mathrm{w} / \mathrm{cm} 2$ associado à técnica de drenagem linfática manual. 0 grupo 2 (G2) foi submetido ao ultrassom com frequência padronizada em 3 Mhz no modo contínuo e intensidade variando de $0,86 \mathrm{w} / \mathrm{cm} 2$ a $1,5 \mathrm{w} / \mathrm{cm} 2$ associado à técnica de drenagem linfática manual e aplicação do Therapy Taping®. Para verificar o efeito do Therapy Taping® na Lipodistrofia, a aplicação foi realizada em forma de "teia", por meio do direcionamento da bandagem no sentido do retorno linfático mostradas nas Figuras $\mathbf{1}$ e $\mathbf{2}$.

Para aplicação do ultrassom e do Therapy Taping ${ }^{\circledR}$, os indivíduos foram posicionados em decúbito dorsal e em semiflexão de joelho. Para aplicação da drenagem linfática na região abdominal os indivíduos foram posicionados em decúbito dorsal e em semiflexão de joelho e para aplicação da técnica nos flancos foram posicionados em decúbito lateral, em semiflexão de joelho e quadril.

O ultrassom Sonopulse, da Ibramed, com gel La Vertuan, foi aplicado em seis quadrantes, quatro abdominais (lateral direito superior e inferior e lateral esquerdo superior e inferior) e flancos. A drenagem linfática manual foi realizada na região abdominal e flancos, assim como a aplicação do Therapy Taping ${ }^{\circledR}$.

\section{Resultados}

$\infty \times \infty \times \infty \times \infty \times \infty \times \infty \times \infty \times \infty \times \infty \times \infty \times \infty \times \infty \infty$

Participaram do estudo seis indivíduos do sexo feminino, divididos em dois grupos, com três integrantes em cada. As características dos grupos foram descritas na Tabela 1. No G1 uma das integrantes teve duas gestações; a média da idade foi de 24 anos; a média de peso foi de $83,70 \mathrm{~kg}$ e a média da altura foi de $168 \mathrm{~cm}$. Os participantes foram submetidos ao ultrassom associado à drenagem linfática manual. No G2 uma das integrantes teve uma gestação; a média da idade foi de 22 anos; a média de peso foi de 75,70 kg e a média da altura foi de $164 \mathrm{~cm}$. Os participantes submetidos ao ultrassom associado à drenagem linfática manual e ao Therapy Taping ${ }^{\circledR}$, mostrados na Tabela 1.

Por meio do protocolo aplicado, foi constatado que não houve perda significativa de peso corporal, sendo o peso pós-protocolo apresentado com média de $500 \mathrm{~g}$ em ambos os grupos. As medidas foram recolhidas no início e no final da aplicação do estudo, na região da cicatriz onfálica, três centímetros acima e três centímetros abaixo. Foram observados resultados superiores no G2 quando comparados com o G1 nas reduções em centímetros da região da cicatriz onfálica e três centímetros acima, já a três centímetro abaixo o G1 apresentou redução superior ao G2, porém quando comparadas as somatórias de redução em todas as áreas entre grupos observou-se que a redução em centímetros foi a mesma, totalizando $29 \mathrm{~cm}$, de acordo com a Tabela 2.

Quando comparados os resultados de redução por meio de porcentagem, o G2 apresentou redução superior apenas em área de cicatriz onfálica, o que é destacado no Gráfico 1.

\section{Discussão}

$\infty \times \infty \times \infty \times \infty \times \infty \times \infty \times \infty \times \infty \times \infty \times \infty \times \infty \times \infty \times \infty$

Participaram do estudo apenas indivíduos do sexo feminino com circunferência abdominal 
Jéssica Lopes de Souza, Pedro Vinicius Nabeiros Martins, Ricardo Mingorance, Márcia Regina Pinez Mendes e Laila Moussa

acima de 80 centímetros, sendo este um quesito para risco de doenças cardiovasculares. 0 aumento da circunferência abdominal é um problema observado, hoje, em escala nacional. Segundo Pititto ${ }^{\mathbf{1 4}}$, pesquisas vêm mostrando que o acúmulo de gordura abdominal, mesmo em não obesos, está associado a doenças metabólicas e cardiovasculares comuns na meia-idade. Assim, torna-se evidente que a morbidade, a mortalidade, bem como as alterações metabólicas nos obesos estão mais relacionadas à quantidade de tecido adiposo depositado no abdome. Conforme Caires $^{15}$, a maioria da população busca terapia física, nutricional ou medicamentosa como recursos fundamentais no processo terapêutico, que visa à redução do perímetro abdominal.

O ultrassom e o gel de acoplamento com princípios ativos são ferramentas largamente utilizadas para tratamento da lipodistrofia. Segundo Jesus et al. $^{16}$ dentre os efeitos promovidos pela terapia baseada no ultrassom, a fonoforese consiste na veiculação de princípios ativos (substâncias farmacológicas) para dentro das diferentes camadas do tecido biológico. Esse método torna-se vantajoso ao conduzir os agentes farmacológicos para estruturas profundas da pele e do tecido subcutâneo de uma maneira segura, inodora e fácil, minimizando a probabilidade de efeitos colaterais

0 estudo teve como objetivo comparar os efeitos do ultrassom associado à drenagem linfática manual com o ultrassom associado à drenagem linfática manual e ao Therapy Taping ${ }^{\circledR}$ na redução da circunferência abdominal. Ambos os grupos apresentaram redução da circunferência, porém com ausência de significância para comparação da eficácia das técnicas entre eles.

Como comprovado na literatura, o ultrassom associado à drenagem linfática manual são métodos eficazes na área de fisioterapia dermatofuncional. 0 estudo de Silvestre et al., ${ }^{17}$ por exemplo, que associou o ultrassom com frequência padronizada de $3 \mathrm{MHz}$, modo contínuo e dose de $1,2 \mathrm{~W} / \mathrm{cm} 2$, com a drenagem linfática manual de Leduc, demonstrou que o método é eficaz, pois o ultrassom melhorou a circulação e favoreceu as trocas metabólicas.

Em relação ao método Therapy Taping ${ }^{\circledR}$, não há estudos do tipo experimental intervencionista que comprovem o resultado da técnica na redução da circunferência abdominal, impossibilitando comparações.

A aplicação da bandagem foi realizada antes do início do protocolo, na região do antebraço, para identificar possíveis reações alérgicas. Ass participantes permaneceram com a bandagem por três dias, não apresentando manifestações alérgicas. Porém, de acordo com o criador do método, a aplicação deveria ser realizada previamente na região do estudo (abdominal e flancos), pois de acordo com Junior ${ }^{13}$, tanto no momento da indicação da bandagem quanto ao longo do tratamento, devem ser observadas algumas áreas com mais atenção, para não desenvolver irritação na pele, em razão de serem mais finas e sensíveis, dentre as quais está a região abdominal.

A ausência da dieta sensorial, recomendada pelo criador da técnica devido à escassez do período, pode ter influenciado nos resultados por ser um possível fator que gerou manifestações alérgicas a duas das participantes na oitava sessão, com características de rubor e prurido, havendo a necessidade de interrupção da aplicação do Therapy Taping ${ }^{\circledR}$. As participantes finalizaram 0 tratamento apenas com o ultrassom e drenagem linfática manual, impossibilitando a comparação proposta inicialmente.

De acordo com os resultados obtidos, pode-se concluir que a aplicação do ultrassom associado à drenagem linfática manual tem resultados positivos na redução da circunferência abdominal, porém, devido às intercorrências, não foi possível afırmar a eficiência do Therapy Taping ${ }^{\circledR}$ para o objetivo proposto. Sugere-se, portanto, a realização de novas pesquisas com maior período de aplicação do protocolo, maior número de participantes e com as recomendações propostas pelo criador da técnica Therapy Taping ${ }^{\circledR}$

\section{Conclusão}

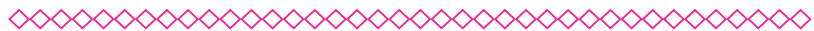

A partir dos resultados apresentados nesta pesquisa, concluiu-se que os resultados obtidos com o Therapy Taping ${ }^{\circledR}$ não influenciaram significativamente na redução da circunferência abdominal das participantes. Deve-se levar em 
conta que duas participantes componentes do G2 apresentaram reação alérgica ao material do Therapy Taping, sendo assim, a aplicação do método foi suspensa do protocolo na oitava sessão para ambas as participantes, que continuaram com o tratamento utilizando o ultrassom associado à técnica de drenagem linfática manual até finalizar a $12^{\mathrm{a}}$ sessão. É de essencial importância relatar que devido à escassez do período disponível para realização da pesquisa, as participantes não realizaram a dieta sensorial proposta pelo criador da técnica, necessária em modo avançado.

A amostra foi realizada em um número pequeno de participantes e com um tempo limitado, o que pode ter influenciado nos resultados das comparações, sendo assim, propõe-se que sejam realizados novos estudos com amostras em maior número e com maior extensão da aplicação do protocolo proposto.

Vale salientar que, mediante pesquisas realizadas, não foram encontrados estudos do tipo experimental intervencionista com o tema proposto, sendo este um trabalho inovador, abrindo um campo de ideias para composição de novos estudos.

\section{Referências}

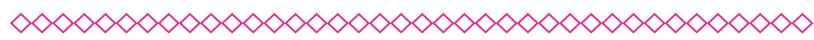

1. Meyer PF, Cavalcante JL, Ronzio AO, Silva RMV, Medeiros ML, Patricio RA, et al. Efeitos das Ondas Sônicas de Baixa Frequência no Fibro Edema Gelóide: Estudo de Caso. Rev. Bras. Terap. e Saúde. 2011; 1 (2): 31-36.

2. Borges FS. Dermato-Funcional: Modalidades Terapêuticas nas Disfunções Estéticas. 2 ed. São Paulo: Phorte Ltda.; 2010.

3. Miliani GB, João SMA, Farah ED. Fundamentos da Fisioterapia Dermato-Funcional: Revisão de Literatura. Fisioterapia e Pesquisa. 2006; 13 (1): 37-43.

4. Pitanga FJG, Lessa I. Indicadores Antropométricos de Obesidade como Instrumento de Triagem para Risco Coronariano Elevado em Adultos na Cidade de Salvador - Bahia. Arq. Bras. Cardiol. 2005 Jul; 85 (1): 26-31.
5. Silva RMV, Costa LS, Silva ACF, Cesar AL, Ramos MLVS, Cruz DRL et al. Aplicação do Ultrassom Terapêutico com Fins Lipolíticos em Pacientes Portadores de Adiposidade Localizada. Fisioterapia Ser. 7 (2), 2012.

6. Ricardo GD, Caldeira GV, Corso ACT. Prevalência de Sobrepeso e Obesidade e Indicadores de Adiposidade Central em Escolares de Santa Catarina, Brasil. Rev. bras. epidemiol. 2009 Set; 12 (3): 424435.

7. Godoy-Matos AF, Oliveira J, Guedes EP, Carraro L, Lopes AC, Mancini MC, et al. Diretrizes Brasileiras de Obesidade 3. ed. São Paulo: AC Farmacêutica; 2009.

8. Neves SR, Oliveira D. Eficácia da Associação de Técnicas Manuais e Eletrotermoterapia na Redução de Medidas do Abdome. Rev Biol e Saúde da UNISEP. 2007;1(1):67-71.

9. Guirro E, Guirro R. Fisioterapia DermatoFuncional: Fundamentos, Recursos e Patologias. 3. ed. São Paulo: Manole; 2002.

10. Bassalobre M, Altomare M, Oliveira JTM. Drenagem Linfática de Abdome Pré e PósCirurgia de Lipoabdominoplastia: Análise por Linfocintilografia. Fisioterapia Ser. 1 (4), 2006.

11. Costa CT, Rocha JVC. Eficácia da Drenagem Linfática no Linfedema Pós-Traumático de Membros Inferiores - Estudo de Caso. Ciências biológicas e da saúde. 2015; 2 (2): 424-35.

12. Therapy Taping Association [homepage na internet] Método Therapy Taping [acesso em 03 out. 2015]. Disponivel em: http://www.taping.com.br/ metodo/.

13. Junior NM. Bandagem Terapêutica - Conceito de Estimulação Tegumentar 1 ed. São Paulo: Roca; 2015 .

14. Pitito BA. Efeitos um programa de Intervenção no Estilo de Vida sobre o perfil de risco cardiometabólico de uma população nipo-brasileira de alto risco cardiovascular. [Tese de Doutorado]. São Paulo: Universidade de São Paulo, 2009. 
15. Caires NFR et al. Sobrepeso e obesidade entre funcionários da Universidade Estadual de Feira de Santana. Rev. Baiana Saúde Pública. 2005; 29(2): 238-50.

16. Jesus GS, Ferreira As, Mendonça AC. Fonoforese x Permeação Cutânea. Fisioter Mov. 2006;19(4):83-8.

17. Silvestre CP, Zanon CS. O uso do ultra-som associado com a drenagem linfática manual no tratamento do fibro edema gelóide.Ágora: R. Divulg. Cient. 2009; 16 (2) 


\section{Anexos}

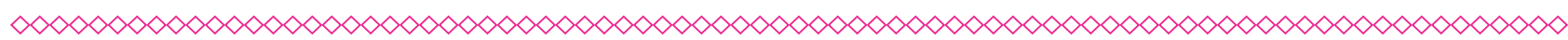

Figuras 1 e 2 : Aplicação do Método Therapy Taping ${ }^{\circledR}$

Figura 1

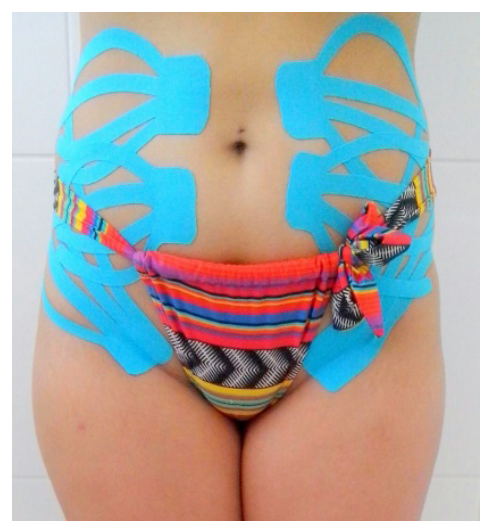

Figura 2

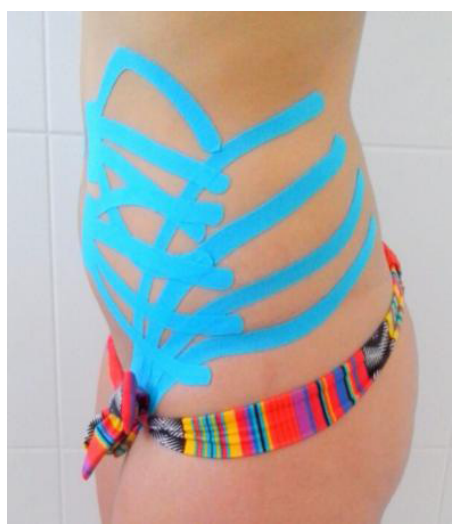

(clique para voltar ao texto)

Tabela 1: Características dos grupos 1 e 2.

\begin{tabular}{|c|c|c|}
\hline \multicolumn{4}{|c|}{ Tabela 1 - Características dos Grupos } \\
\hline Características & Grupo 1 & Grupo 2 \\
\hline Média de Idade (anos) & 24,66 & 22,33 \\
\hline Média de Peso (kg) & 83,7 & 75,7 \\
\hline Média de Altura (cm) & 168 & 164 \\
\hline $\mathrm{N}^{\circ}$ de integrantes que tiveram gestaçס̄es & 1 & 1 \\
\hline
\end{tabular}

(clique para voltar ao texto)

Tabela 2: Perimetria e redução total da circunferência abdominal

\begin{tabular}{|c|c|c|c|c|}
\hline \multicolumn{5}{|c|}{ Grupo 1} \\
\hline Áreas de Aplicação & $\begin{array}{c}\text { Cicatriz } \\
\text { Onfálica }\end{array}$ & $\begin{array}{c}3 \mathrm{~cm} \\
\text { Acima }\end{array}$ & $\begin{array}{c}3 \mathrm{~cm} \\
\text { Abaixo }\end{array}$ & Somatória \\
\hline Pré Protocolo & 292 & 275 & 304 & 871 \\
\hline Pós Protocolo & 284 & 264 & 294 & 842 \\
\hline Redução Total & 8 & 11 & 10 & 29 \\
\hline \multicolumn{5}{|c|}{ Grupo 2} \\
\hline Áreas de Aplicação & $\begin{array}{l}\text { Cicatriz } \\
\text { Onfálica }\end{array}$ & $\begin{array}{c}3 \mathrm{~cm} \\
\text { Acima }\end{array}$ & $\begin{array}{c}3 \mathrm{~cm} \\
\text { Abaixo }\end{array}$ & Somatória \\
\hline Pré Protocolo & 296 & 275 & 317 & 888 \\
\hline Pós Protocolo & 285 & 263 & 311 & 859 \\
\hline Redução Total & 11 & 12 & 6 & 29 \\
\hline
\end{tabular}

(clique para voltar ao texto) 


\section{Gráfico 1: Redução da circunferência abdominal}

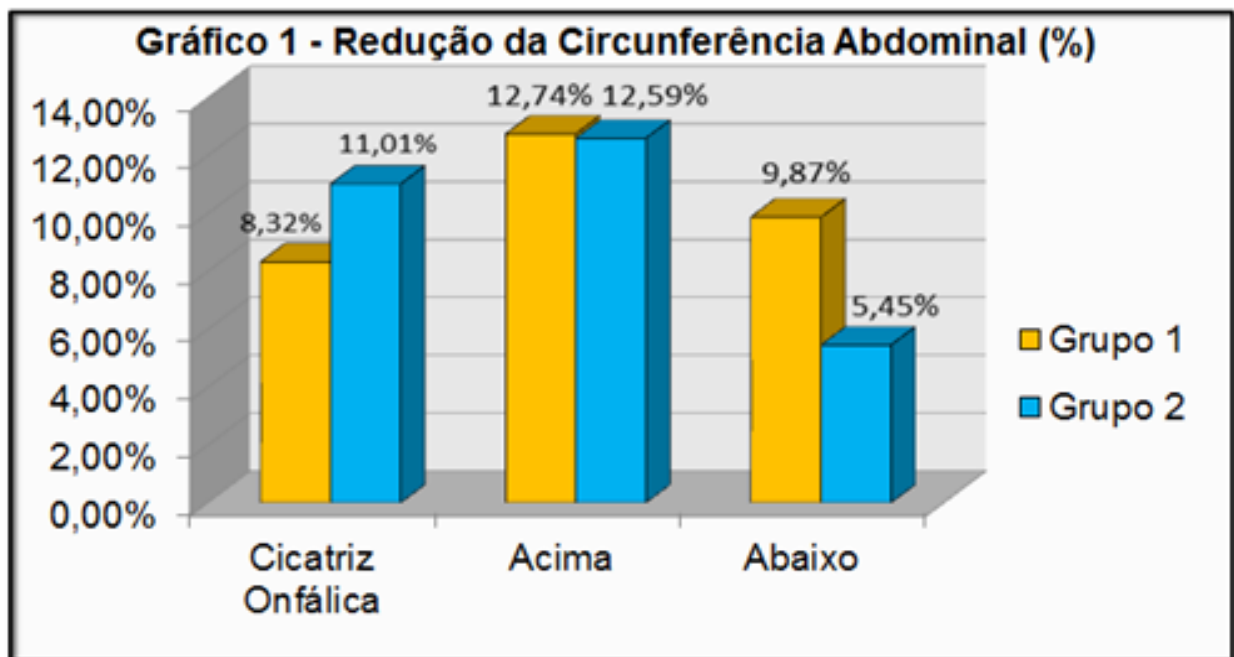

(clique para voltar ao texto) 